\title{
Recent Developments in Compound Semiconductor Microwave Power Transistor Technology
}

\author{
Christopher M. Snowden \\ Filtronic plc, Salts Mill Road, Shipley, BD18 3TT UK \\ and School of Electronic and Electrical Engineering, The University of Leeds, LS2 9JT UK. \\ Email: christopher.snowden@filtronic.com
}

\begin{abstract}
This paper addresses the state-of-the-art in microwave and millimetre-wave power transistor technology. The relative performance of microwave power transistor technology from $1 \mathrm{GHz}$ to $60 \mathrm{GHz}$ is reviewed. The fundamental technological drivers in the design of microwave compound semiconductor power transistors are discussed as the basis for developing optimum designs. A new high yield power GaAs pHEMT process is described and the application of this technology to multi-carrier microwave power amplifiers is discussed, achieving state-of-the-art performance, with output powers of up to $120 \mathrm{~W}$ with $70 \%$ efficiency at $2.1 \mathrm{GHz}$.
\end{abstract}

\subsection{INTRODUCTION}

Solid-state microwave power amplifiers are required to deliver RF power levels between a few Watts at millimeter-wave frequencies to peak powers of over a kilowatt at $1 \mathrm{GHz}$, in communications and Radar applications. The design of high performance microwave power amplifiers for future communications systems requires very low levels of distortion, high efficiencies and the ability to handle multiple carriers whilst simultaneously operating at very low levels of intermodulation distortion. The performance of the power amplifier depends critically upon the choice of active device and the embedding circuit design. Recent high efficiency designs, require power transistors with very high cut-off frequencies and very low 'on' resistances. This has led to an upsurge of interest in using compound semiconductor 
transistors, and in particular pseudomorphic high electron mobility transistors (pHEMTs) and heterojunction bipolar transistors (HBTs), which can achieve higher efficiencies and improved linearity at these frequencies [1,2].

\subsection{POWER TRANSISTOR PERFORMANCE}

Choice of Technology. The choice of power transistor for a given application strongly depends on the mode of operation at the required frequency. Key performance parameters are the power output and power added efficiency, which in turn relate to the maximum operating voltage and current handling capability of the transistor and its large-signal gain. A comparison of the power output and power added efficiency (PAE) performance of a variety of popular RF technologies is shown in Figure 1 and Figure 2.

In the frequency range $900 \mathrm{MHz}$ to $1.8 \mathrm{GHz} \mathrm{LDMOS}$ has the lowest output power density (power per gate/emitter periphery), whilst the HBT has the highest power density. This apparent potential size advantage of HBTs is partially mitigated by the need for ballast resistors and thermal management on the smaller die. LDMOS is well established in both infrastructure and handset applications. Recently, pHEMT and HBTs have achieved significant levels of penetration into new designs of handset power amplifier, offering improved efficiency. PHEMT (and MESFET) driver transistors are often used in existing designs to drive LDMOS output stages in wireless infrastructure designs, where until recently the power handling and cost of LDMOS was superior to that of other technologies. There have also been significant advances in new generations of LDMOS technology [3] aimed at improving the efficiency and linearity. 
Cellular infrastructure base station power amplifiers represent the largest market for high power microwave transistors. Although LDMOS FETs are the most widely used technology today in this application below $2 \mathrm{GHz}$, their relatively low unity gain cut-off frequency (usually below $7 \mathrm{GHz}$ ), means that they struggle to simultaneously meet the efficiency and linearity requirements for third-generation mobile communication systems operating above 2 GHz. Over the past three years very high power GaAs MESFETs, HFETs and PHEMTs have demonstrated superior performance to LDMOS at these frequencies $[4,5,6]$. Until recently the cost of compound semiconductor transistors has been significantly higher than that of LDMOS. However, with a target price of less than $\$ 1 /$ Watt for a packaged part, recent developments in manufacturing technology (notably 6" GaAs wafers) promise competitive pricing from compound semiconductor devices. A new high power, high yield GaAs FET technology is described in this paper that satisfies the demanding performance required for third-generation mobile communication power amplifiers. The cost of packaging remains a substantial element of the final cost of discrete power transistors and this has led to the development of higher levels of integration in multi-chip modular designs.

Other applications, such as Radar, have used both silicon bipolar and LDMOS devices at lower frequencies (up to $4 \mathrm{GHz}$ ), but increasingly GaAs-based transistors (MESFETs, HEMTs, HBTs) are being used to improve performance in these systems. Above $8 \mathrm{GHz}$, these GaAs transistors are used exclusively at this time. Increased interest in electronically-scanned (Escan) radars has led to the requirement for high efficiency power amplifier monolithic microwave integrated circuits (MMICs). An 8 Watt, $10 \mathrm{GHz}$ power amplifier for Escan radar applications is shown in Figure 3, which was fabricated using the process described later in this paper. At frequencies above $18 \mathrm{GHz}$, where power levels of few Watts are usually required in applications such as Point-to-Point transceivers, HEMT MMIC technology is dominant. 
Wide-bandgap Semiconductors. The recent introduction of wide-bandgap semiconductors such as $\mathrm{SiC}$ and $\mathrm{GaN}$ has led to the development of new types of power transistor with very high breakdown voltages and the potential for operating voltages above $40 \mathrm{~V}$. These devices achieve very high power densities - GaN holds the record of $9.2 \mathrm{~W} / \mathrm{mm}$ of gate periphery at $8 \mathrm{GHz}[7,8]$. The breakdown field in $\mathrm{SiC}$ and $\mathrm{GaN}$ is over five times higher than that in $\mathrm{GaAs}$, compensating for the lower mobility of these materials. Hence, although the knee voltage of these transistors is higher than that of GaAs FETs (often $4 \mathrm{~V}$ compared with $1 \mathrm{~V}$ ), the higher operating voltage allows power densities of up to an order of magnitude higher than that achievable with GaAs or LDMOS. These transistors also offer favourable thermal properties with better conductivity and tolerance to higher temperatures. AlGaN/GaN heterojunctions allow GaN HEMTs to achieve higher current densities than comparable SiC FETs. However, there are still several technological hurdles to overcome before these technologies mature to the point of providing a cost-competitive reliable alternative to GaAs and $\mathrm{Si}$. In particular, the challenges in growing large $\mathrm{SiC}$ substrates and the absence of a bulk GaN substrate require technologies such as GaN epitaxy on $\mathrm{SiC}$ or Si wafers. At present the relatively small wafer size ( $\leq 3$ " diameter), the high defect density and high levels of traps in these wafers are much worse than that achievable in GaAs, limiting the yield and performance.

\subsection{MICROWAVE POWER TRANSISTOR DESIGN}

Fundamental Parameters. The discussion in the remainder of this paper will focus on compound semiconductor FETs and the development of a new GaAs pHEMT power transistor technology that achieves relatively high breakdown voltages, for application in mobile communications and radar. Generally speaking, in most power amplifier designs, the power transistor is required to have a minimum peak drain operating voltage of double the quiescent operating bias voltage. In 
some modes of operation (such as Class E and Class F) this breakdown voltage may have to be up to three times higher than the bias voltage. This provides a lower bound on the breakdown voltage.

It is necessary to recognise that in depletion-mode FETs, with negative gate bias voltages, that the gate-drain peak voltage is usually the limiting factor in terms of breakdown. Although LDMOS, $\mathrm{SiC}$ and $\mathrm{GaN}$ transistors can achieve breakdown voltages of over $60 \mathrm{~V}$ or more, GaAs-based transistors rarely exceed $25 \mathrm{~V}$ for breakdown for $\mathrm{V}_{\mathrm{DSB}}$ or $\mathrm{V}_{\mathrm{DGB}}$ (determined for a given level of gate current in GaAs FETs). This usually limits the DC supply to less than $12 \mathrm{~V}$ for GaAs FETs. In GaAs MESFETs and HEMTs the breakdown voltage is usually determined by the interaction of surface geometry, surface material properties and active channel design. In particular, key parameters are the gate-drain separation, the Schottky gate parameters including the built-in potential, metallization and recess (single or double) and the drain-source channel parameters (doping, material composition and layer thicknesses). Novel GaAs FET structures including gamma gates (the shape of the gate metallization) and field-plate FETs have been reported, which achieve breakdown voltages of over 100V [9]. An experimental gamma-gate structure, developed for a pHEMT is shown in Figure 4. A disadvantage of this approach is that the cut-off frequency of the FET is reduced as a consequence of the increased gate-drain capacitance. A power pHEMT process with a ' $\mathrm{T}$ ' gate structure and exceptionally good DC and microwave performance is described later in this paper.

A key parameter in power FET design is the breakdown field, which limits the peak electric field between the gate and drain, Table 1. The breakdown field is a strong function of the material quality and the density of impurities (including the donors). Over the past ten years improvements in GaAs material technology have led to an increase in the breakdown fields achievable in practical structures and these now approach the theoretical limit. 
The breakdown voltage in a GaAs power FET is maximised by careful design of the channel, whilst employing double recess gate structures and appropriate passivation of the semiconductor surface. The gate-drain spacing is optimized to maximize the breakdown voltage, whilst avoiding excessive drain channel resistance. Surface states in GaAs and InP give rise to surface depletion, which interacts with passivation layers causing unwanted surface leakage, breakdown, and transient trapping phenomena that can manifest themselves as gate and drain lag effects.

The drain current (with a maximum value $\mathrm{I}_{\max }$ at $\left.\mathrm{V}_{\text {knee }}\right)$, knee voltage $\left(\mathrm{V}_{\text {knee }}\right)$ and gain in microwave FETs is directly dependent on the electron mobility, velocity and electron density in the conducting channel. GaAs has a mobility, which is over four times higher than that of Si and achieves very high peak velocity over a short distance (velocity overshoot). Hence, in short gate length GaAs-based power FETs the maximum current handling capacity per unit periphery is the highest of all the semiconductor technologies considered here. Unfortunately, the mobility and velocity decrease as the doping density increases (ie. as the current handling increases). This problem can be overcome using in GaAs pHEMT structures with undoped channels and separate highly doped supply layer (normally separated with a thin undoped spacer layer). However, the desire for higher charge densities in the supply levels has to be balanced against the requirement to maximize breakdown voltage, which also decreases with increasing doping level (due to impact or avalanche ionisation).

Finally, it is important to consider the voltage swing required on both the gate and drain. In addition to the limits imposed on the drain voltage by the knee voltage and the breakdown voltage, it is necessary to consider the impact of the pinch-off voltage and gate conduction on the gate voltage swing. Recently there has been a move towards circuit designers preferring enhancement mode devices (e-mode), which only require positive gate bias voltages. These devices tend to have a higher breakdown voltage and high gain, although the positive pinch-off 
voltage $\mathrm{V}_{\text {po }}$ coupled with the gate barrier height restrict the useful voltage swing to usually less than $0.5 \mathrm{~V}$ and gate-leakage currents may be problematic in some designs. In contrast a depletion-mode (d-mode) device or quasi-emode $\left(\mathrm{V}_{\mathrm{po}} \sim-0.5 \mathrm{~V}\right)$, allow larger gate voltage swings and reduced gate leakage. The pinch-off voltage itself is a function of charge density (doping) and epitaxial channel layer thickness. The MESFET and HEMT, like other FETs, are charge-control devices, where the gate charge and gate capacitance are a strong non-linear functions of gate potential. The charge-control mechanism, together with the drain and source bias determines the drain-source current. The non-linear behaviour of the capacitances can have a significant impact on the operation of power amplifiers under large-signal conditions and must be considered during the design of the transistor and its embedding circuit.

\subsection{ELECTRO-THERMAL MODELLING AND DESIGN}

The performance of new power transistor designs can be assessed using physical device models in conjunction with time domain simulators to study directly large-signal behaviour $[10,11]$, including intermodulation distortion (within the limitations of lengthy time-domain simulation for this type of signal) [14]. During the past ten years, a fast class of simulation tool known as a 'quasi-two-dimensional (Q2D) model', has allowed relatively accurate results to be obtained in a small fraction of the time consumed by traditional two-dimensional models. The microwave power FET development described here has made use of the thermally self-consistent Leeds Physical Model (LPM), which is a fast fully physical Q2D simulator, with the capability of providing large signal time-domain data and allowing CAD timescale simulations $[11,12,13,14]$.

A typical power pHEMT structure investigated in this work is shown in Figure 5. In these devices, many of the layers in the device structure are very thin $(<100 \AA)$ and often form 
heterostructure interfaces where quantization of the electron energy occurs, such as in the FET channel. The LPM model uses a self-consistent quantum mechanical model to represent the gate charge-control model [13]. In addition to its role in the full device model, this charge-control model has been used to investigate the epitaxial layer design. An example of a conduction energyband diagram for a pHEMT epitaxial structure is shown in Figure 6. This clearly shows the 'rectangular' well, characteristic of InGaAs pHEMTs, showing the quantized electron energy levels. This profile has been designed to minimize parasitic conduction between the gate metal and the channel (the 'well').

The strong temperature dependence of electron mobility and relatively poor thermal conductivity of GaAs requires an accurate thermal model that can be coupled self-consistently to the electrical model of the transistor. Thermal modeling in this work has utilized a dynamic self-consistent thermal impedance matrix model of non-linear heat flow for the full power FET die $[10,14,16]$, supported by infra-red measurements. A simulated temperature profile, from the models used in this work, for a cross-section through a power FET is shown in Figure 7, where peaks in the temperature correspond with the regions in the FET channel at the drain edge of the gate fingers. It has been shown that detailed device structure, including the device contact finger layout, surface metallisation and vias, all impact on the transient thermal response [16]. Also, thermal time constants can vary by several orders of magnitude, depending on the scale of power dissipation and temperature sensitive areas and volumes. This analysis provides valuable insight for thermal design.

Equivalent circuit modeling, based on extraction of model parameters from measured data, plays a key role in characterizing the devices and process. Passive elements, including via holes (used to ground the source contacts in the power FETs), have been characterized using this technique. Nonlinear model elements based on extraction from multi-frequency, bias-dependent $\mathrm{S}$ parameter measurements form the basis of the FET intrinsic equivalent circuit model. 


\subsection{A VERY HIGH PERFORMANCE POWER FET TECHNOLOGY}

A high yield power pHEMT technology, based on an InGaAs/AlGaAs/GaAs molecular beam epitaxy layer structure, has been developed to support microwave power amplifier applications with single die capable of handling RF powers of up to $75 \mathrm{~W}$. Several novel processes were developed to fabricate these devices on 6" GaAs wafers, including a low resistance 'T' gate structure with silicon nitride $\mathrm{SiN}$ passivation, Figure 8 . Both $0.5 \mu \mathrm{m}$ and $0.25 \mu \mathrm{m}$ gate length versions have been developed, where the ' $\mathrm{T}$ ' gate sits in a dry-etch defined gate recess. The 0.5 $\mu \mathrm{m}$ gate exhibits a gate resistance of less than $38 \Omega / \mathrm{mm}$. Excellent pinch-off uniformity is maintained by a high selectivity etch-stop layer. A specially developed multi-layer surface passivation process allows a high breakdown voltage to be obtained, whilst reducing dispersion in the current-voltage characteristics to a very low level. The finished 6" wafers are thinned (in-situ) to ensure good thermal performance and minimal via hole impedance, Figure 9. Via hole resistances typically lie in the range $2 \mathrm{~m} \Omega$ to $4 \mathrm{~m} \Omega$. The layout of the individual cells is designed to minimize the degradation in gain and power output with increasing scale of the device.

This pHEMT process shows no degradation in breakdown voltage with scaling of the device from $200 \mu \mathrm{m}$ to $150 \mathrm{~mm}$ gate width. Optimum gate-drain spacing, balancing the trade-off between breakdown voltage and parasitic drain resistance, coupled with careful design of the epitaxial layer allows gate-drain breakdown voltages of over $40 \mathrm{~V}$, a high $\mathrm{I}_{\max }$ and a low 'on' resistance to be obtained. Output power densities of $0.75 \mathrm{~W} / \mathrm{mm}$ and drain efficiencies routinely above $70 \%$ have been measured for large gate periphery devices. Yields of over $80 \%$ have been obtained from this process for large power die. Figure 10 shows a finished $60 \mathrm{~mm}$ die (approximately $4 \times 1 \mathrm{~mm}$ ).

This power process supports high breakdown voltages $\left(\mathrm{V}_{\mathrm{DGB}} \sim 40 \mathrm{~V}\right)$, high $\mathrm{I}_{\max }(\sim 450$ $\mathrm{mA} / \mathrm{mm})$, high large-signal gain $\left(\mathrm{G}_{1 \mathrm{~dB}} \sim 16 \mathrm{~dB}\right.$ at $\left.2 \mathrm{GHz}\right)$, and a high cut-off frequency $\mathrm{f}_{\mathrm{T}}\left(\mathrm{f}_{\mathrm{T}} \sim 20\right.$ 
$\mathrm{GHz}$ ) with total gate widths of up to $150 \mathrm{~mm}$. Multi-carrier power amplifiers achieving $18 \mathrm{~W} \mathrm{RF}$ output with an efficiency of $79 \%$ at $1 \mathrm{GHz}$, and 120 Watts with $70 \%$ efficiency at $2.1 \mathrm{GHz}$ have been demonstrated using this technology. A 360 Watt WCDMA power amplifier has been demonstrated achieving over $35 \%$ power added efficiency at $2.1 \mathrm{GHz}$ (compared with $25 \%$ from the state-of-the-art 200W LDMOS WCDMA power amplifier [17]) and demonstrating over 60 $\mathrm{dBc}$ out-of-band spurious signal rejection. The same power transistor design concepts are now being applied to MMIC processes. Two integrated circuit examples, using the 0.5 micron process, are a 50 Watt MMIC achieving 45\% PAE at $2.1 \mathrm{GHz}$ and an X-band MMIC demonstrating 11 Watts from 8.5 to $10.5 \mathrm{GHz}$, with a power added efficiency of over $35 \%$ (slightly larger in area than the version shown in Figure 3). Yields above 85\% were obtained for these power MMICs. In addition to the advantages of higher efficiencies, these pHEMT amplifiers operate with relatively small temperatures rises above ambient, enhancing reliability and reducing heatsinking and cooling requirements. For example, a $96 \mathrm{~mm}$ gate periphery power pHEMT generating $44 \mathrm{~W}$ of RF output power with $66 \%$ efficiency at $2.1 \mathrm{GHz}$, is shown to have a peak surface temperature of $43^{\circ} \mathrm{C}$ in Figure 11.

\section{CONCLUSION}

Significant advances have been made in the development of compound semiconductor power transistors in recent years. A new high breakdown voltage $(\sim 40 \mathrm{~V})$ pHEMT technology, exhibiting state-of-the-art RF and microwave performance has been described, which has properties of low 'on' resistance, high gain, and high cut-off frequencies. This new power process meets the needs of the next generation of wireless infrastructure power amplifier designers and is also well suited to radar applications, demonstrating world-class performance in both hybrid and integrated amplifier designs. 


\section{ACKNOWLEDGEMENTS}

The author acknowledges the contributions of Dr W. Batty, Dr A.J. Panks and the engineers and fabrication staff at Filtronic Compound Semiconductors, Newton Aycliffe.

\section{REFERENCES}

1. I.Takenaka, K.Ishikura, H. Takahashi, K. Asano and M. Kanamori, "Low Distortion High Power GaAs Pseudomorphic Heterojunction FETs for L/S-Band Digital Cellular Base Stations", Proc. IEEE Microwave Theory and Techniques Symposium, pp.1711-1718, June $11-16^{\text {th }}, 2000$.

2. K. Inoue, K.Ebihara, H.Haematsu, T. Igarashi, H.Takahashi and J. Fukaya, “A 240W Push-Pull GaAs Power FET for W-CDMA Base Stations”, Proc. IEEE Microwave Theory and Techniques Symposium, pp. 1719-1722, June 11-16 ${ }^{\text {th }}, 2000$.

3. A. Litwin, Q. Chen, J. Johansson, G. Ma, L.A. Olofsson, P.Perugupalli, "High Power LDMOS Technology for Wireless Infrastructure”, Proc. GAAS 2001, London, pp.61-64, 2001.

4. K. Ebihara, K. Inoue, H. Haematsu, F. Yamaki, H. Takahashi, J. Fukaya, “An ultra broad-band 300W GaAs power FET for W-CDMA base stations", Proc. IEEE Microwave Theory and Techniques Symposium, pp. 649-652, June 2001

5. K. Matsunaja, H. Shimawaki, “A 90W S-Band high power amplifiers for broadband wireless applications", Proc. IEEE Microwave Theory and Techniques Symposium, pp. pp. 73-76, June 2003.

6. J. Staadinger, R. Sherman, T. Quach, M. Miller, L. Fry, “A 15 Watt PEP GaAs pHEMT MMIC power amplifier for 3G wireless transmitters", Proc. IEEE Microwave Theory and Techniques Symposium, pp. 617-620, June 2002 
7. R.J. Trew, "AlGaN/GaN HFET amplifier performance and limitations", Proc. IEEE Microwave Theory and Techniques Symposium, pp. 1811-1814, June 2002

8. Y.F. Wu, D. Kapolnek, J.P. Ibbetson, P.Pariku, B. Keller, U.K. Mishra, "Very high power density AlGaN/GaN HEMTs" IEEE Trans. Electron Dev., Vol. 48, pp.586-590, March 2001

9. A. Wakejima, K. Ota, K. Matsunaga, W. Contrata, M. Kuzuhara, "Field-Modulating plate (FP) InGaP MESFET with high breakdown voltage and low distortion", Proc. IEEE Radio Frequency Integrated Circuits Symposium, , pp. 151-157, June 2001

10. C.M. Snowden, "Large-Signal Microwave Characterization of AlGaAs/GaAs HBTs based on a Physics-Based Electro-Thermal Model", IEEE Microwave Theory and Techniques, Vol.45, No.1, pp. 58-71, January 1997.

11. C.M. Snowden and R. R. Pantoja, "Quasi-two-dimensional MESFET simulations for CAD," IEEE Trans. Electron Devices, vol. 36, pp. 1564-1574, 1989.

12. C.G. Morton, J. S. Atherton, C. M. Snowden, R. D. Pollard and M. J. Howes, "A largesignal physical HEMT model," Proc. IEEE Microwave Theory and Techniques Symposium, pp. 1759-1762, 1996.

13. R. Drury and C.M. Snowden, "A Quasi-Two-Dimensional HEMT Model for Microwave CAD Applications", IEEE Transactions on Electron Devices, pp. 1026-1032, June 1995

14. W. Batty, A. J. Panks, R. G. Johnson and C. M. Snowden, "Electro-thermal modelling of monolithic and hybrid microwave and millimeter wave IC's," VLSI Design, Vol. 10, no. 4, pp. 355-389, 2000.

15. P.J. Rudge, R.E. Miles, M.B. Steer, and C.M. Snowden, "Investigation into Intermodulation Distortion in HEMTs Using a Quasi-2D Physical Model”, IEEE Transactions on Microwave Theory and Techniques, Vol. 49, No.12, pp.2315-2321, December 2001 . 
16. W. Batty, W., C.E. Christoffersen, A.J. Panks, S. David, C.M. Snowden, M.B. Steer "Electro-thermal CAD of power devices and circuits with fully physical time-dependent compact thermal modelling of complex non linear 3-D systems", IEEE Transactions on Components and Packaging Technology, pp. 566-590, September 2001.

17. C. Dragon, W. Brakensiek, D. Burdeax, W. Burger, G. Funk, M. Hurst, D. Rice, “200 W push-pull and $110 \mathrm{~W}$ single-ended high performance RF-LDMOS transistors for WCDMA basestation applications", Proc. IEEE Microwave Theory and Techniques Symposium, pp. 69-72, June 2003 


\section{Figure Captions}

Figure 1. Microwave transistor power output as a function of frequency.

Figure 2 Power added efficiency as a function of frequency for transistor amplifiers.

Figure 3 An 8 Watt $10 \mathrm{GHz}$ Power Amplifier GaAs PHEMT MMIC, 4.5×3 mm.

Figure 4. A cross-section of a gamma-gate HEMT structure, designed to increase breakdown voltage.

Table 1 Semiconductor material parameters.

Figure 5. Power pHEMT cross-sectional structure, showing epitaxial layers and doublerecessed gate.

Figure 6. Conduction band profile of a power pHEMT $\left(\mathrm{V}_{\mathrm{GS}}=0.0 \mathrm{~V}\right)$, showing quantization of the electron energy levels in the rectangular well.

Figure 7. Temperature profile for a cross-section of a power pHEMT.

Figure 8. Cross-section of a power FET showing ' $T$ ' gate details and passivation.

Figure 9. Cross-sections of (a) a thinned power FET showing semiconductor layers supported on gold metallization (b) low resistance via hole.

Figure 10. A 60mm gate width GaAs power FET die (approximately $1 \times 4 \mathrm{~mm}$ ). 
Figure 11. Infra-red image of a $96 \mathrm{~mm}$ gate width power pHEMT operating in a $2.1 \mathrm{GHz}$ power amplifier generating $44 \mathrm{~W}$ of RF at $66 \%$ efficiency. 
Figure 1

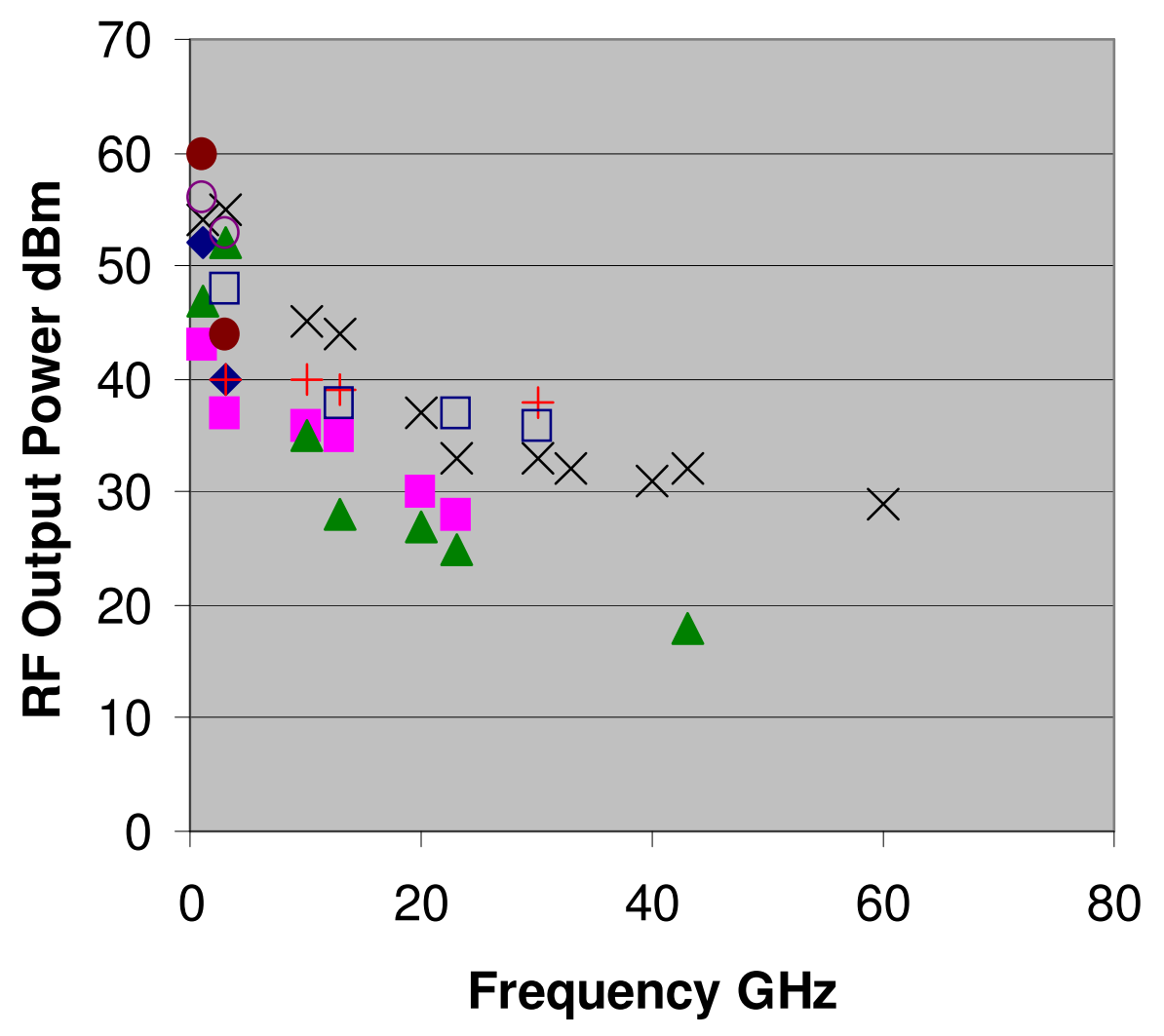

- Si BJT $\mathrm{HBT}$

$\triangle$ GaAs MESFET $\times$ HEMT/HFET

O LDMOS $-\mathrm{SIC}$ SIT

+ AIGaN/GaN HEMT $\square$ SiC MESFET 
Figure 2

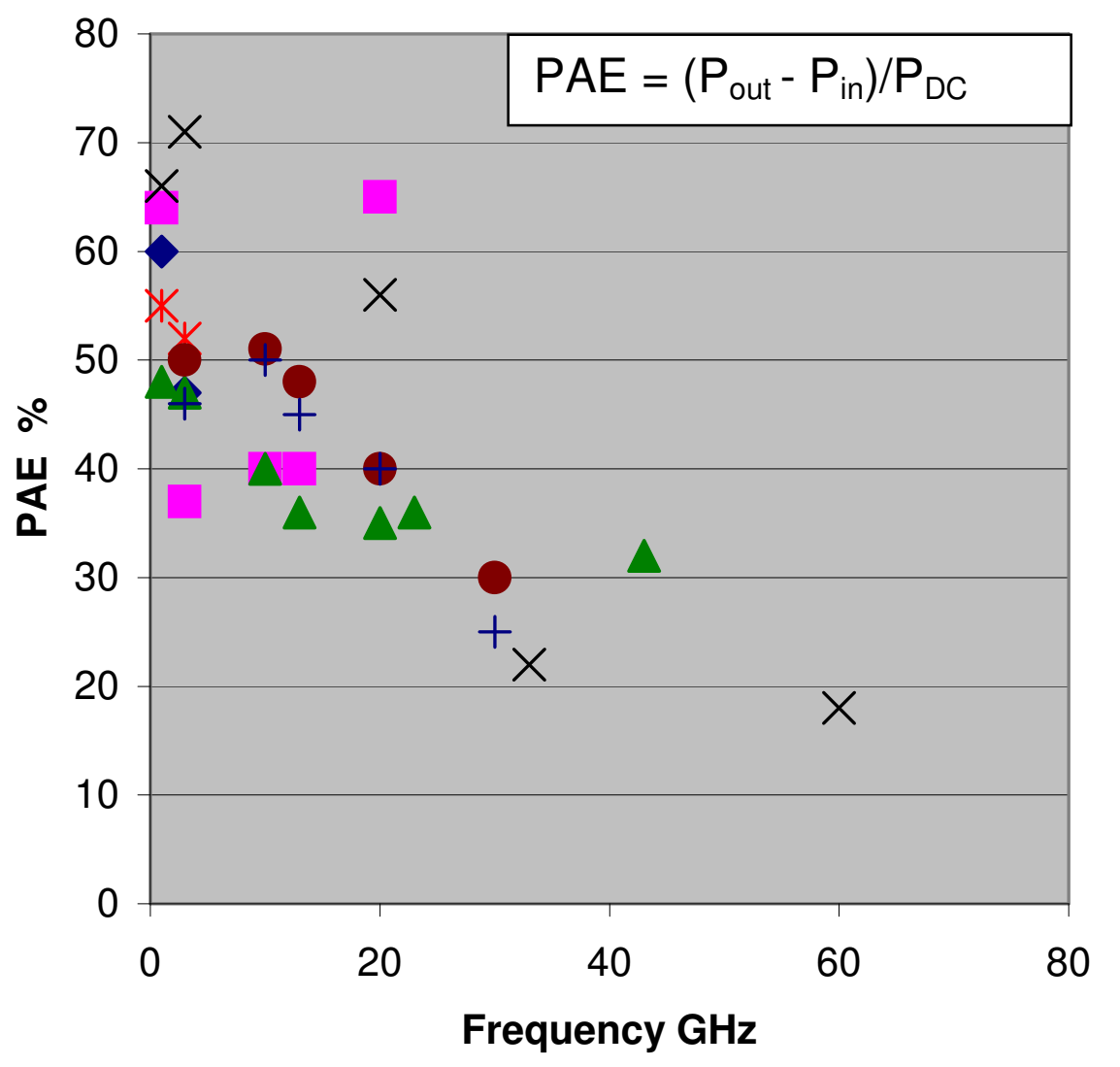

$\diamond \mathrm{Si} \mathrm{BJT} \quad \mathrm{HBT}$

$\triangle$ MESFET $\quad \times$ HEMT/HFET

*LDMOS AIGaN/GaN HEMT

+ SiC MESFET 
Figure 3

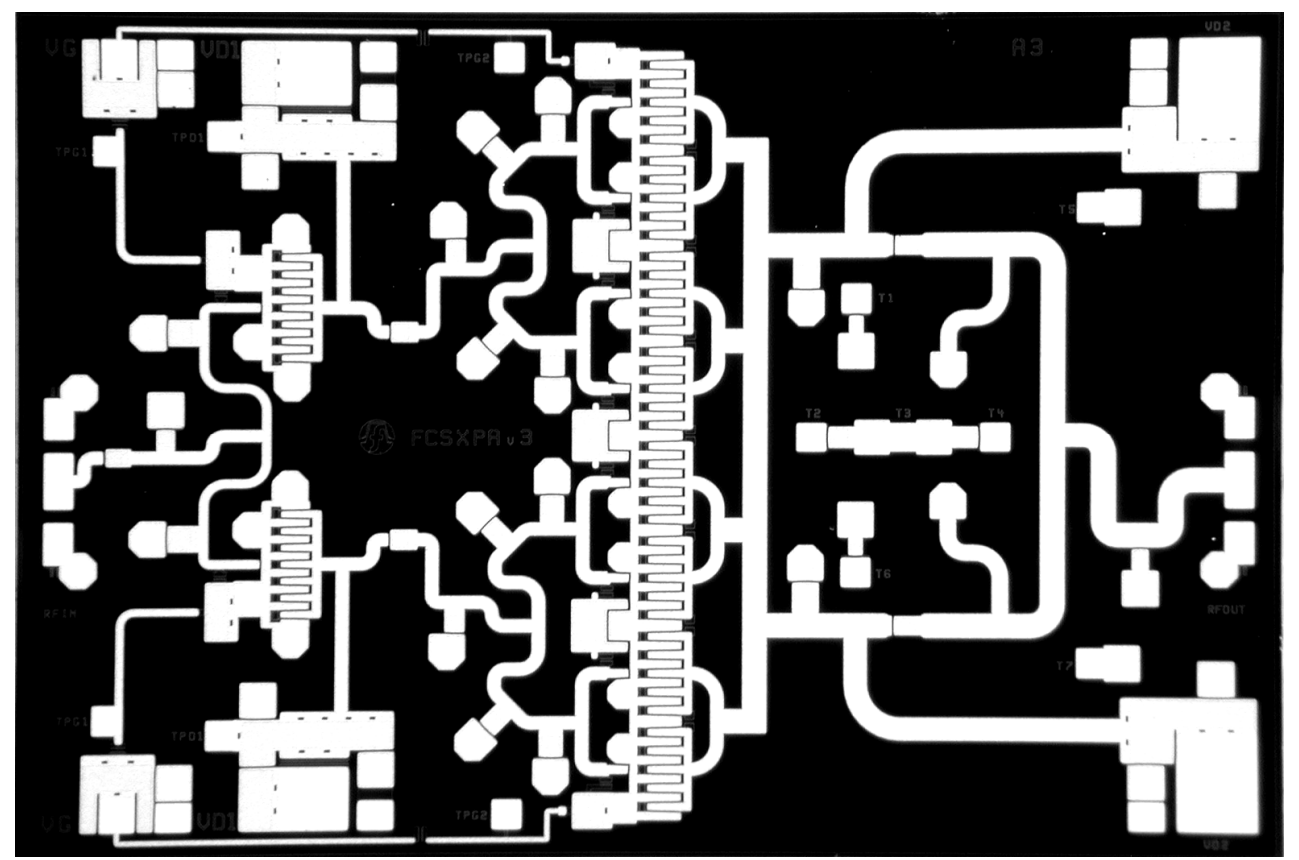


Table 1

\begin{tabular}{|l|cccc|}
\hline & $\mathrm{Si}$ & $\mathrm{GaAs}$ & $\mathrm{SiC}(4 \mathrm{H})$ & $\mathrm{GaN}$ \\
\hline Bandgap (eV) & 1.12 & 1.43 & 3.2 & 3.3 \\
Relative Dielectric Constant & 11.8 & 12.8 & 9.7 & 9 \\
Breakdown Field $\mathrm{MVm}^{-1}$ & 60 & 63 & 350 & 350 \\
Thermal Conductivity $\mathrm{Wm}^{-1} \mathrm{~K}^{-1}$ & 150 & 46 & 490 & 170 \\
Electron Mobility $\mathrm{m}^{2} \mathrm{~V}^{-1} \mathrm{~s}^{-1}$ & 0.135 & 0.6 & 0.08 & 0.1 \\
Saturated Velocity $\times 10^{5} \mathrm{~ms}^{-1}$ & 1 & 1 & 2 & 1.5 \\
Peak Velocity $\times 10^{5} \mathrm{~ms}^{-1}$ & 1 & 7 & 2 & $>2$ \\
\hline
\end{tabular}


Figure 4.

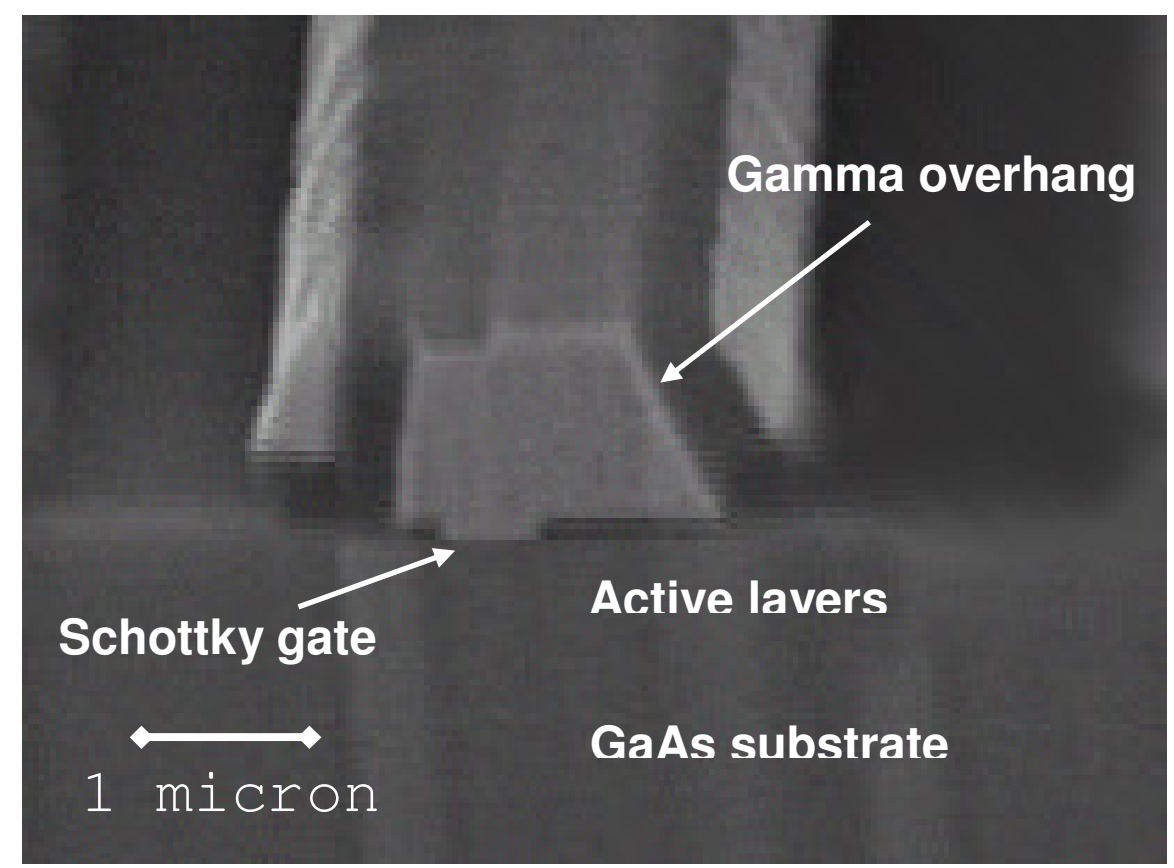


Figure 5.

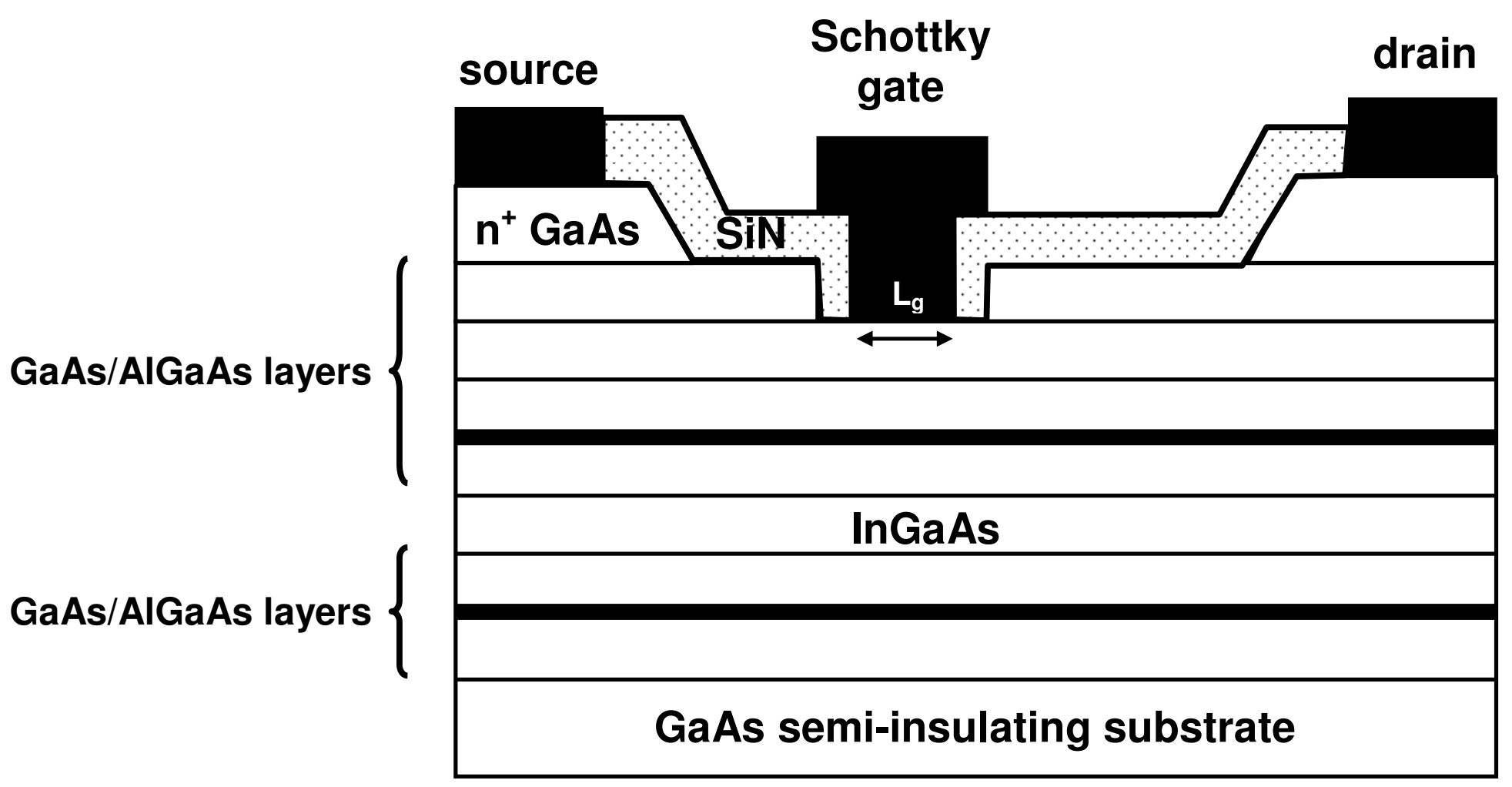


Figure 6

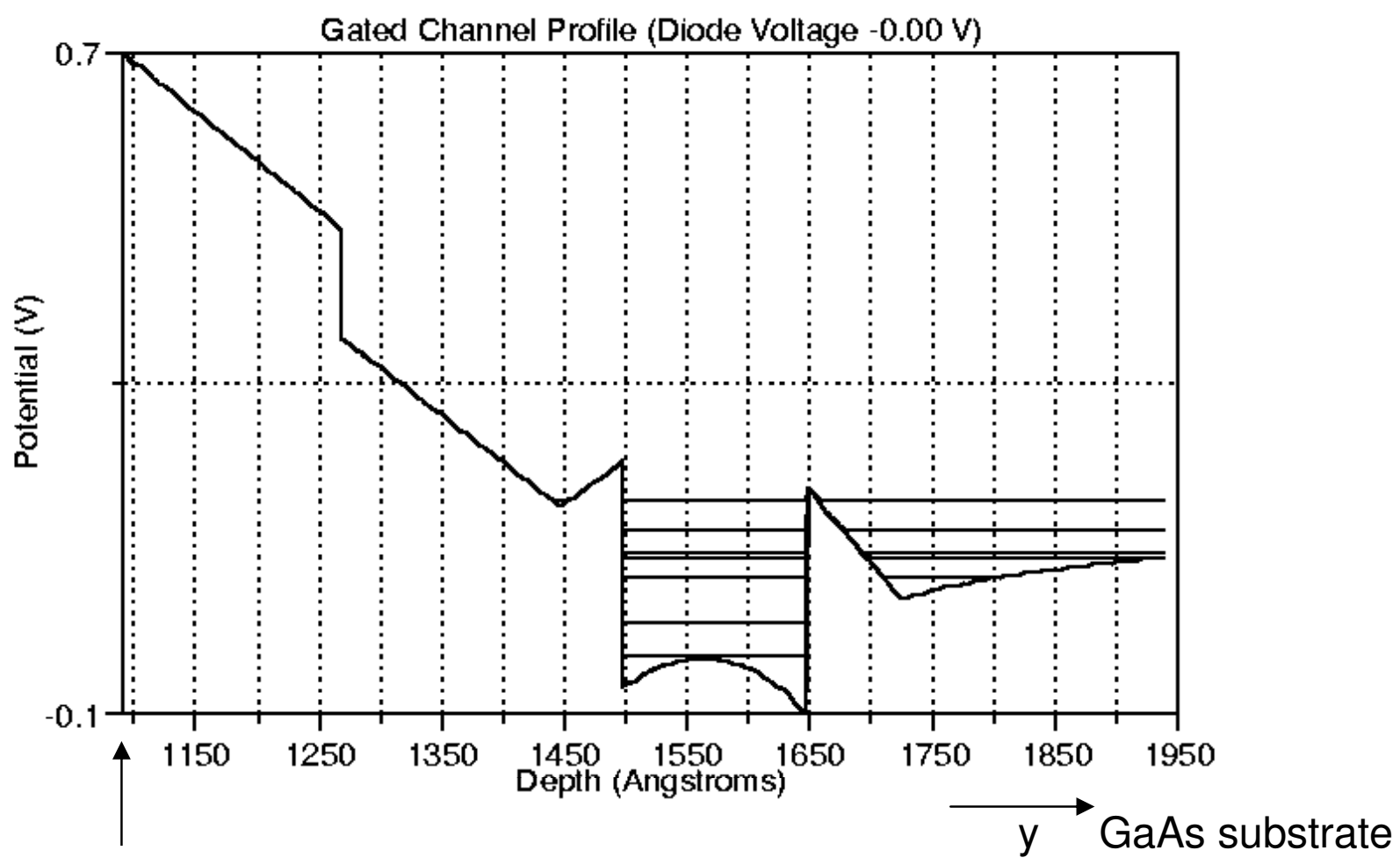

Gate metal location 
Figure 7.

HEMT cross-section
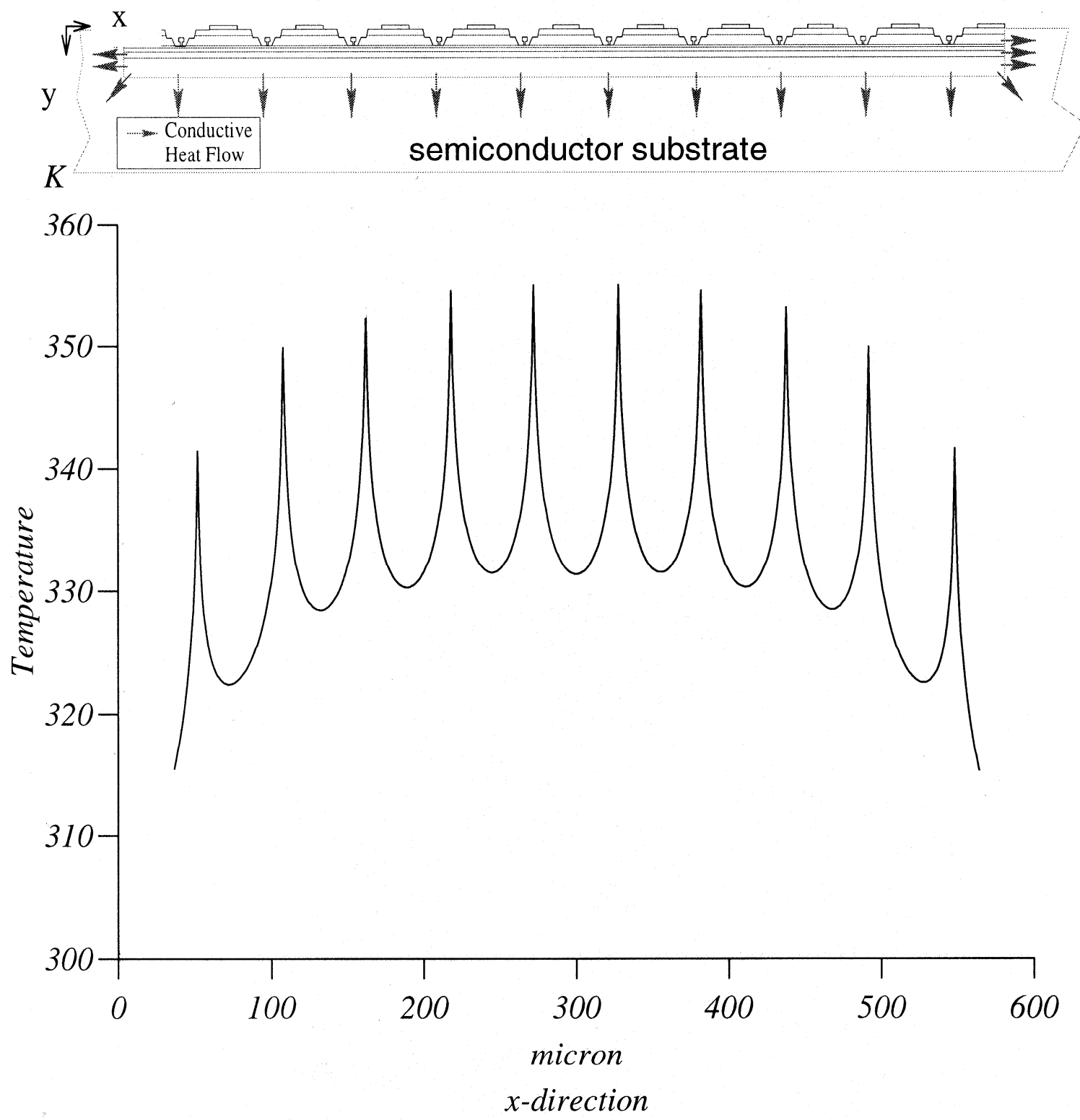
Figure 8.

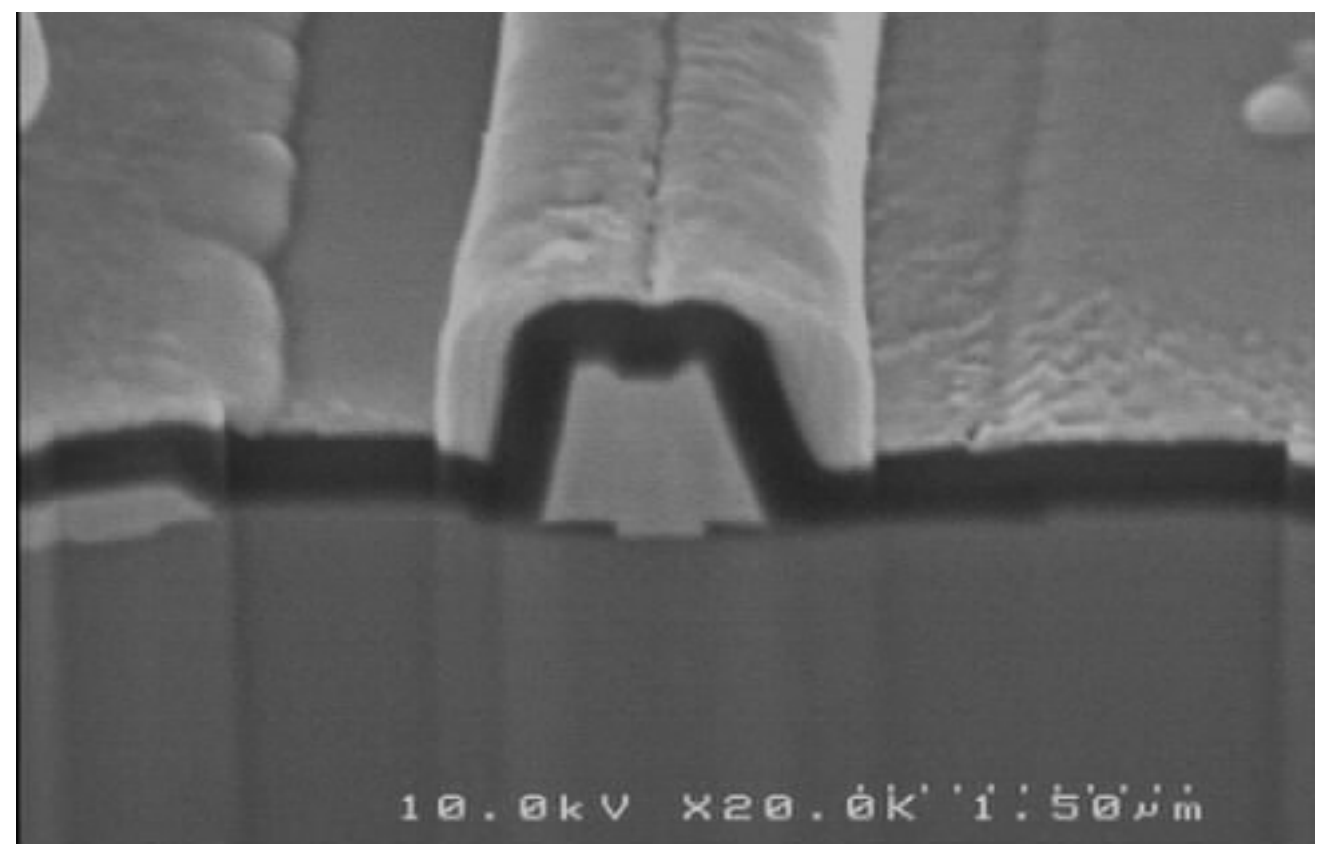


Figure 9.

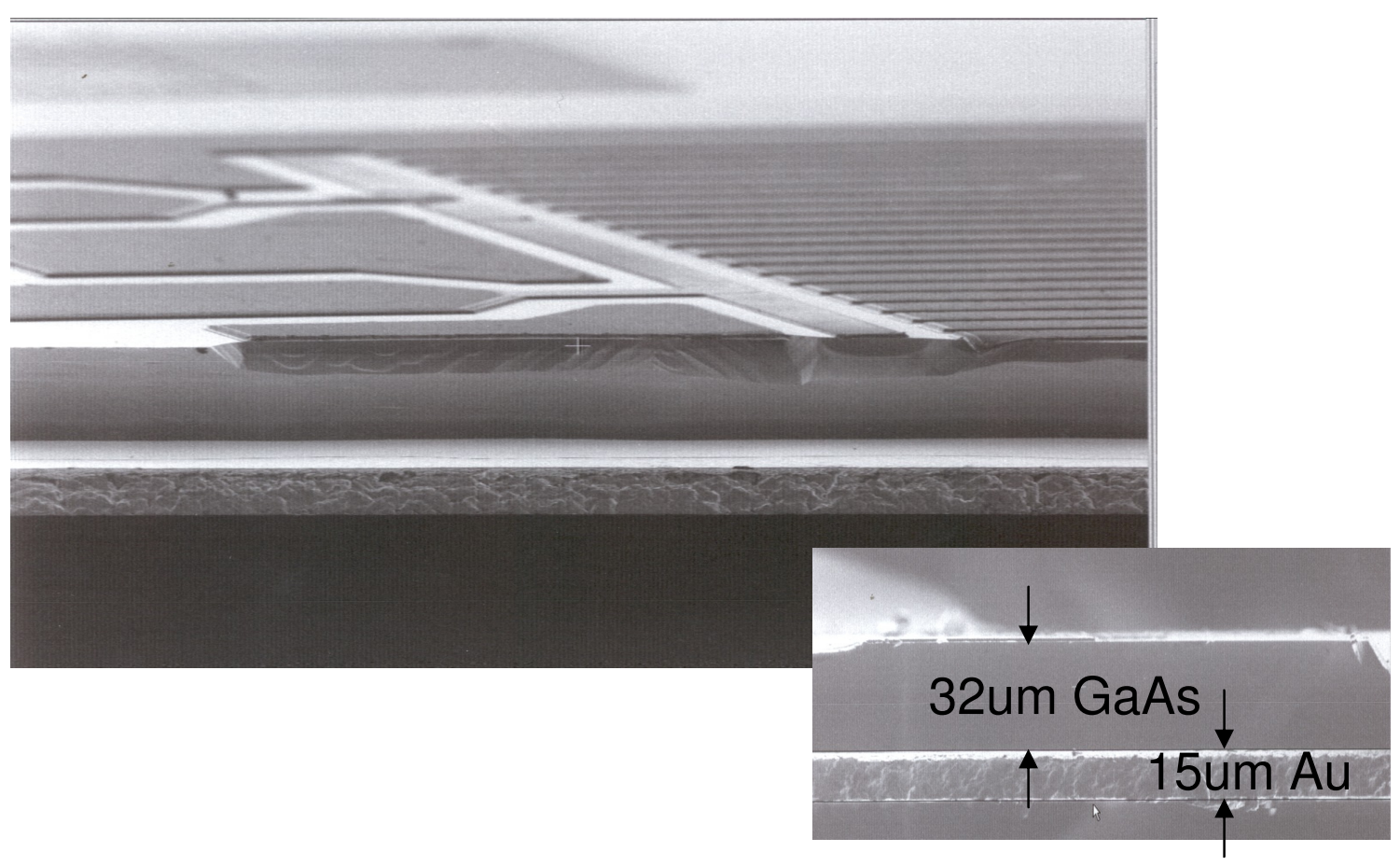

(a)

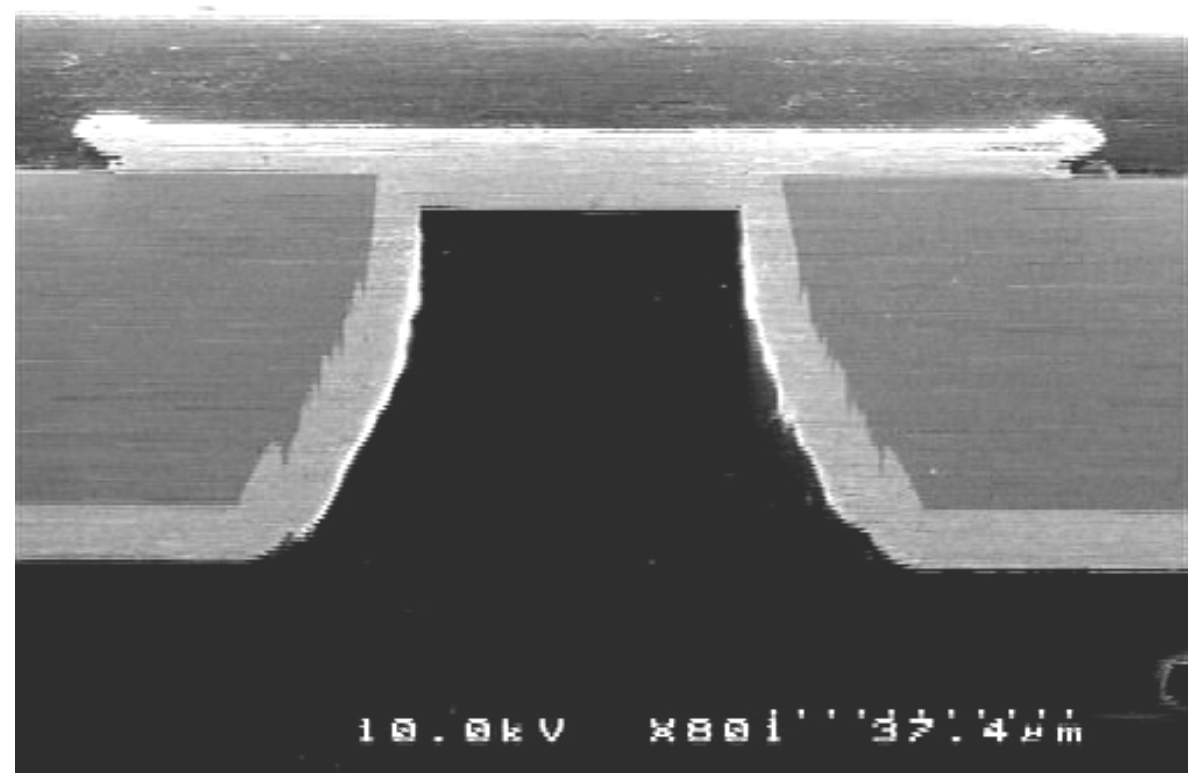

(b) 
Figure 10.

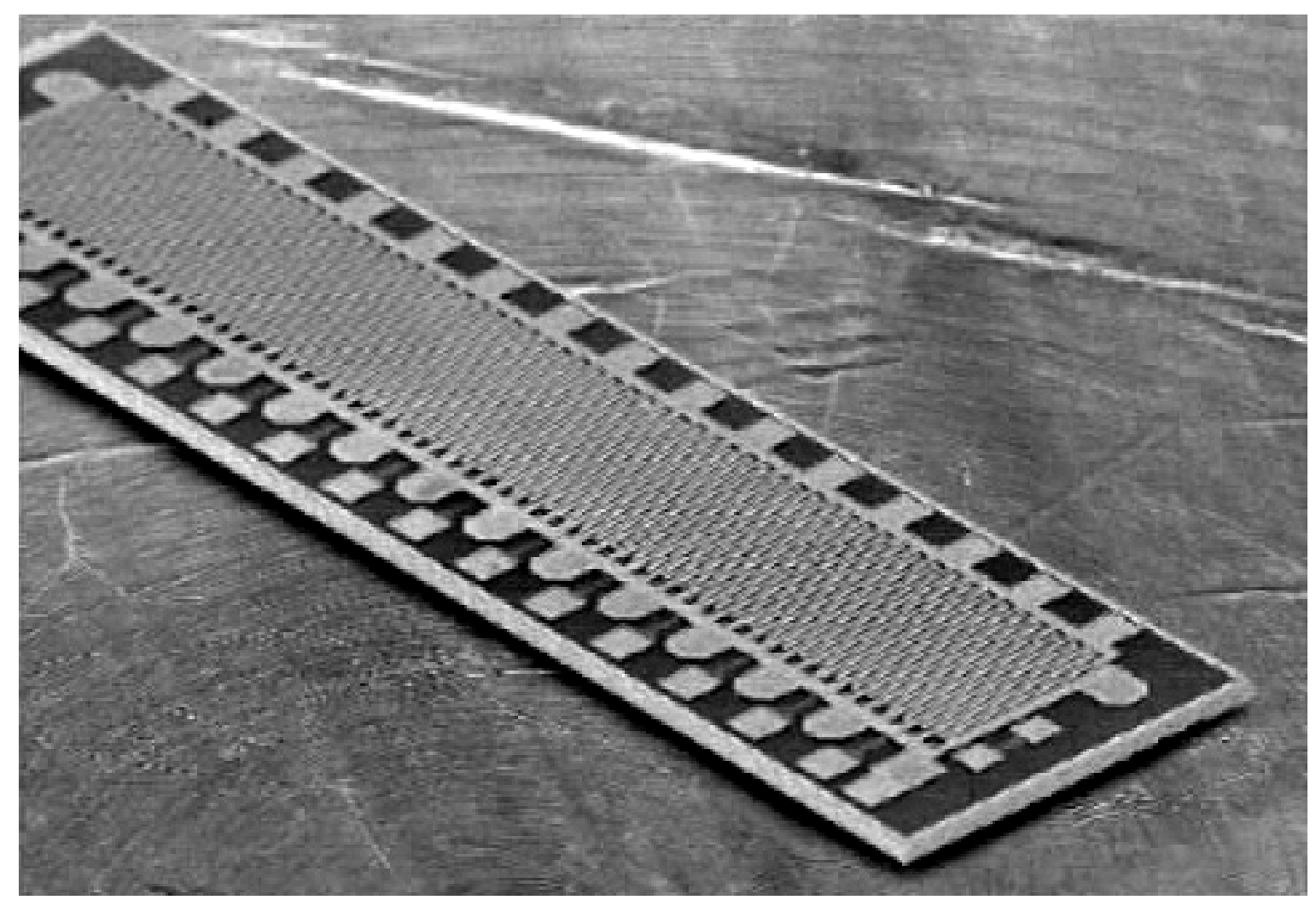


Figure 11.

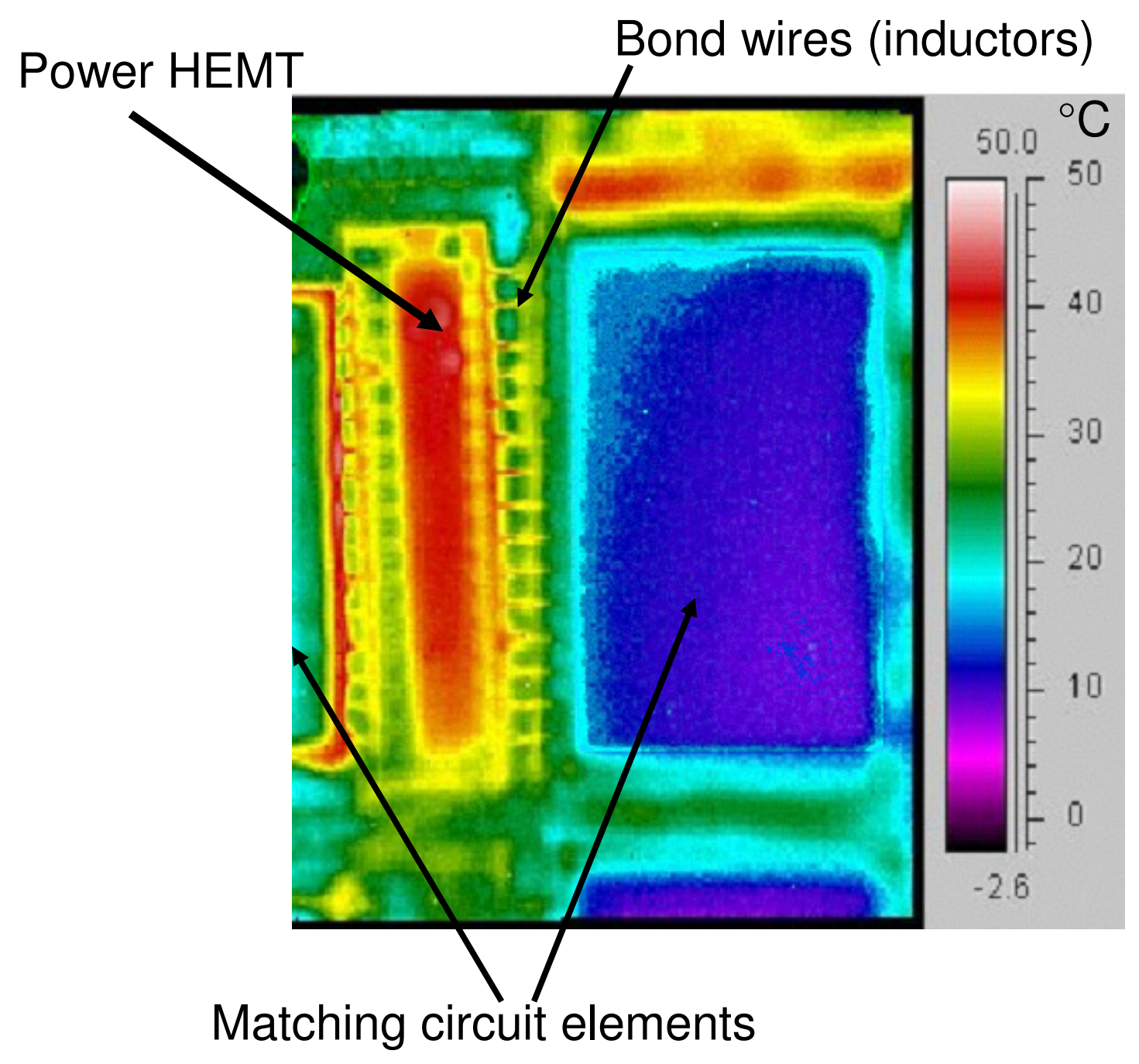


